\title{
Study Day of Food and Water Consumption
}

National Cancer Institute

\section{Source}

National Cancer Institute. Study Day of Food and Water Consumption. NCI Thesaurus.

Code C119828.

The study day that the food and water consumption data is captured. 